\title{
Compact Quad-Band Bandpass Filter Based on Stub-Loaded Resonators
}

\author{
Bal S. Virdee ${ }^{2}$, Mohamad Farhat ${ }^{1}$, and Muhammad Riaz ${ }^{2}$ \\ ${ }^{1}$ London Metropolitan University, Center for Communications Technology, 166-220 Holloway Rd., \\ Holloway, London N7 8DB, United Kingdom \\ ${ }^{2}$ American College of the Middle East, Block 3, Building 1, Egaila Area, Kuwait
}

\begin{abstract}
This paper presents a planar quad-band bandpass filter with high out-of-band rejection. The filter is based on intercoupled stub-loaded resonators, where pairs of resonators are electromagnetically coupled to each other and the feedlines. This results in excitation of passbands, where the first and the third passbands are generated by $\lambda / 4$ resonators. The second and the fourth passbands are excited by $\lambda / 2$ resonators. The proposed technique provides sufficient degree of freedom to control the center frequency and bandwidth of the four passbands. In addition, the five transmission zeros created around the passbands results in a quad-band filter with high selectivity, sharp $3 \mathrm{~dB}$ cut-off frequency, high isolation, and low passband insertion-loss. The proposed technique was verified practically. Design methodology and experimental results of the prototype filter are provided.
\end{abstract}

Index Terms-Quad-band, stub-loaded resonators, microstrip filter, planer filter

\section{INTRODUCTION}

The growing demands of wireless communication applications necessitates RF transceivers to operate in multiple but separated frequency bands so that users can access various services, such as WLAN, IEEE 802.16 (WiMAX) and RFID, with a single multimode handset or terminal. Higher order bandpass filters are routinely constituted by cascading multiple single-/dual-mode resonators. This straightforward approach unfortunately increases the overall filter size and raises the fabrication cost to a great extent. Hence, there is a demand for quad-band filters that are small enough to be easily integrated inside RF transceivers. Other salient characteristics sought after in quad-band filters are low insertion-loss, excellent passband selectivity in order to efficiently utilize the EM spectrum, and wide stopband or outof-band rejection to minimize EMI. These requirements present a challenge for the design engineers. Until now, only few methods have been reported on quad-band bandpass filters [1]-[5].

In [1], the quad-band filter is realized by using negative refractive-index transmission-line (NRI-TL) metamaterials. The filter was constructed on a substrate with relative dielectric constant of 10.2, a thickness of $1.27 \mathrm{~mm}$, and a dielectric loss tangent of 0.002 . Each of the four passbands can be controlled to some degree by varying the parameters of the NRI-TL. However, the design parameters are too complex and the circuit size is of $0.26 \lambda_{\mathrm{g}} \times 0.52 \lambda_{\mathrm{g}}$.

To reduce the circuit size, the quad-band filter using CPWfed dual-mode double-square ring resonators was proposed in [2]. Resonant frequencies can be controlled by tuning the perimeter ratio of the square rings. The filter was fabricated on Rogers $\mathrm{RO} 3003$ substrate with dielectric constant 3, loss tangent of 0.0013 , and thickness of $1.57 \mathrm{~mm}$. It has dimension of $0.43 \lambda_{\mathrm{g}} \times 0.043 \lambda_{\mathrm{g}}$. The bandwidth of the four passbands is different and their loss varies from 2.18-0.93 dB. The quadband filter in [3] is implemented by loading a transmissionline with either open or short-circuited stubs in a parallel configuration. With this structure the passband response deteriorates by loading the transmission-line resulting from the complex coupling between adjacent resonant stubs. This technique requires an optimization method to control the center frequency and bandwidth of filter, and was fabricated on a substrate with a dielectric constant of 3.38 and a thickness is $0.508 \mathrm{~mm}$. The dimensions of the filter are $1.09 \lambda_{\mathrm{g}} \times 0.76 \lambda_{\mathrm{g}}$. The quad-band filter in [4] is realized by inserting two stepped-impedance resonators (SIR) within coupling gap formed between the input and output feedlines. The filter's passbands can be individually controllable by modifying the dimensions of the SIRs. Although the filter is miniature in size $\left(0.56 \lambda_{\mathrm{g}} \times 0.04 \lambda_{\mathrm{g}}\right)$, its passbands are of varying bandwidth and unequally spaced, it has a high insertion-loss of $1.6 \mathrm{~dB}$, and its $3 \mathrm{~dB}$ roll-off is skirt not sharp. The filter is fabricated on a substrate with relative permittivity of 2.2 and thickness of $0.8 \mathrm{~mm}$. In [5] a pair of square-ring loaded resonators is used to excite quadruple-modes. The two resonators are connected to each other with a microstrip-line and coupled to the input and output feedlines. To save space the sides of the square ring are meandered. However, the filter's passbands are unequally spaced, and its $3 \mathrm{~dB}$ roll-off skirt is not very sharp. This filter was fabricated on a 2 inch diameter $0.5 \mathrm{~mm}$ thick $\mathrm{MgO}$ wafer of dielectric constant 9.8 with double-sided $\mathrm{YBa}_{2} \mathrm{Cu}_{3} \mathrm{Oy}$ thin films. The filter has a size $0.37 \lambda_{\mathrm{g}} \times 0.45 \lambda_{\mathrm{g}}$. Furthermore, the filter is very expensive to maintain as it needs to be cooled to a temperature of $77 \mathrm{~K}$.

In this Paper, a unique quad-band bandpass filter structure is proposed which is based on inter-coupled $\lambda / 4$ and $\lambda / 2$ stubloaded resonators. The first and third passbands are determined by the $\lambda / 4$ stub-loaded resonator, and the second 
and fourth passbands determined by the $\lambda / 2$ stub-loaded resonator. The proposed quad-band filter generates five transmission zeros located around the passbands to realize high selectivity, sharp $3 \mathrm{~dB}$ roll-off and high out-of-band rejection. The planar structure facilitates the design and reduces fabrication costs. The filter is miniature compared to the aforementioned filters with a size of $0.25 \lambda_{\mathrm{g}} \times 0.3 \lambda_{\mathrm{g}}$, which eases integration in wireless systems. The two pairs of resonators provide sufficient degree of freedom to control the center frequency and bandwidth of the four passband responses. A prototype filter was designed and developed at 2.4/3.5/5.2/6.8 GHz for combination of WLAN (2.4/5.2 GHz), WiMAX (3.5 GHz) and RFID (6.8 GHz) applications.

\section{FILTER CONFIGURATION AND ANALYSIS}

\section{A. Resonators Configuration}

Fig. 1 shows the configuration of the proposed filter. Stubloaded resonators 1 are short-circuited at the coupling ends, and their other ends are parallel coupled to the $50 \Omega$ input/output feedlines of width W2. The ends of stub-loaded resonators 3 and 4 are open-circuited, and are also parallel coupled with the feedlines. The $\lambda / 4$ stub-loaded resonators ( 1 and 2) excite the first and third passbands in the filter response, and the $\lambda / 2$ resonators ( 3 and 4 ) excite the second and fourth passbands.

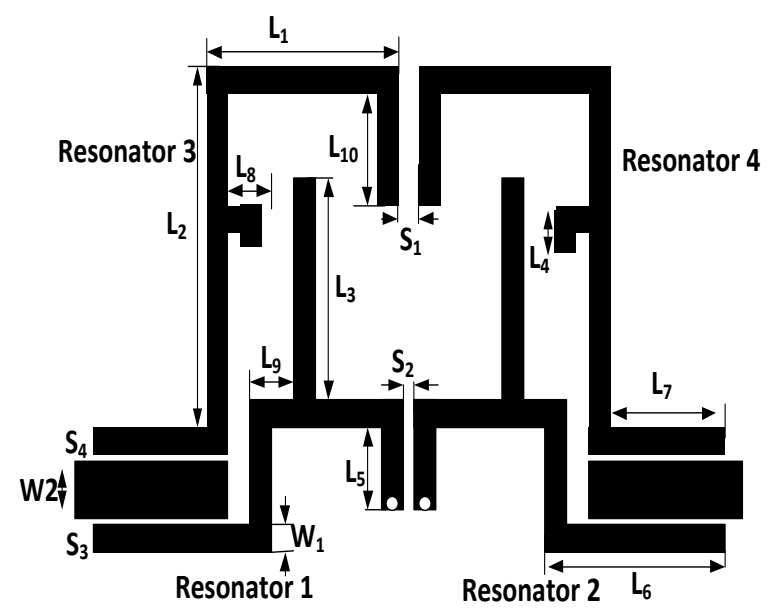

Fig. 1. Layout of the proposed quad-band bandpass filter structure.

\section{B. Analysis of the Quad-Band Bandpass Filter}

The structure of the proposed $\lambda / 2$ and $\lambda / 4$ stub-loaded resonators are shown in Fig. 2(a) and Fig. 3(a), respectively. In Fig. 2(a), the $\lambda / 2$ microstrip resonator is defined by parameters $L_{1}, L_{2}, Z_{1}$ and $Z_{2}$ that denote the length and characteristic impedance of the microstrip-line and open-stub, respectively. Odd and even-mode analysis can be used to characterize the resonator. Fig. 2(b) and 2(c) show the odd and even-mode equivalent circuits, respectively. Odd and even frequencies are given by [6]:

$$
\begin{aligned}
& f_{\text {odd }}=f_{2}=\frac{n c}{2 L_{1} \sqrt{\varepsilon_{\text {eff }}}} \\
& f_{\text {even }}=f_{4}=\frac{n c}{\left(L_{1}+2 L_{2}\right) \sqrt{\varepsilon_{\text {eff }}}}
\end{aligned}
$$

Where $\varepsilon_{\text {eff }}$ is the effective dielectric constant, $n$ is the mode number $(n=1,3,5 \ldots$,$) and c$ is the speed of light in free space $\left(3 \times 10^{8} \mathrm{~m} / \mathrm{s}\right)$.

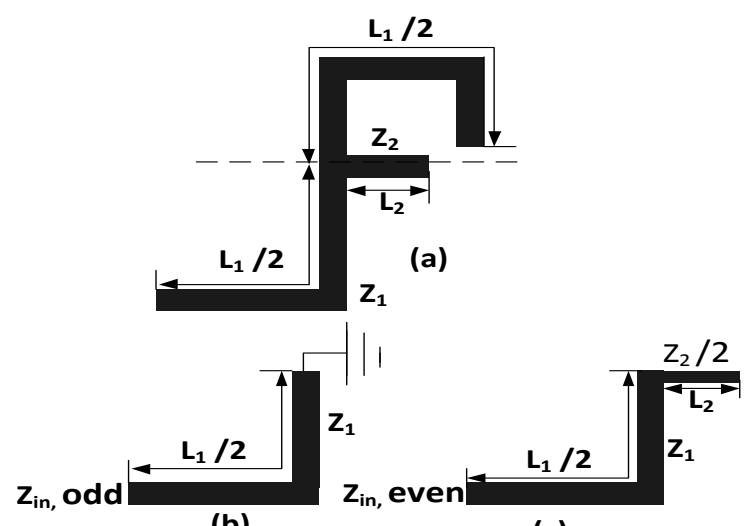

(b)

(c)

Fig. 2 (a) Structure of the proposed $\lambda / 2$ stub-loaded resonator, (b) odd-mode equivalent circuit, and (c) even-mode equivalent circuit.

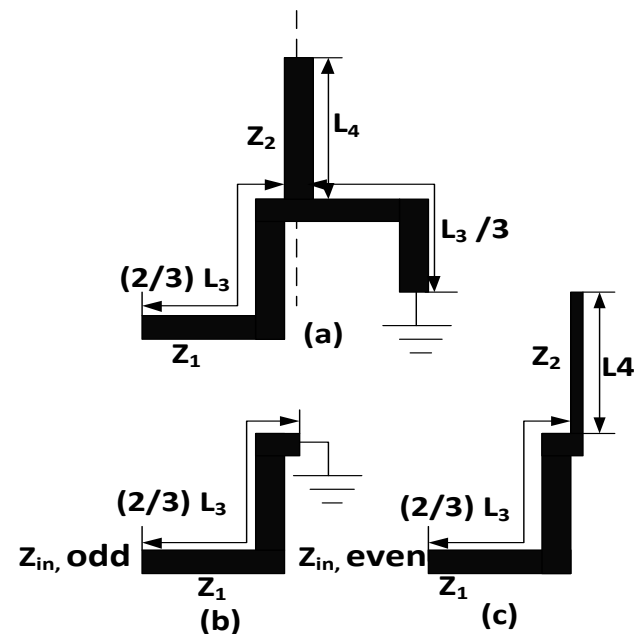

Fig. 3. (a) Structure of the proposed $\lambda / 4$ stub-loaded resonator, (b) odd-mode equivalent circuit, and (c) even-mode equivalent circuit.

The $\lambda / 4$ stub-loaded resonator in Fig. 3(a) is defined by $L_{3}$, $L_{4}, Z_{1}$ and $Z_{2}$ denoting the length and the characteristic impedance of the microstrip-line and open-stub, respectively. The short-circuited stub is shunted and located $2 / 3$ from the bottom of the resonator. Fig. 3(b) and 3(c) show the odd and even-mode equivalent circuit, respectively, of the $\lambda / 4$ stubloaded resonator. The odd and even frequencies are given by: 


$$
\begin{aligned}
& f_{\text {odd }}=f_{1}=\frac{n c}{4 L_{3} \sqrt{\varepsilon_{\text {eff }}}} \\
& f_{\text {even }}=f_{3}=\frac{n c}{\left(2 L_{3}+L_{4}\right) \sqrt{\varepsilon_{\text {eff }}}}
\end{aligned}
$$

\section{SimUlation AND MEASURED REsults}

To demonstrate the practical feasibility of the proposed concept, a quad-band filter was designed for fundamental resonant frequencies at $2.4 / 3.5 / 5.2 / 6.8 \mathrm{GHz}$ for WLAN $(2.4 / 5.2 \mathrm{GHz})$ WiMAX $(3.5 \mathrm{GHz})$ and RFID $(6.8 \mathrm{GHz})$ applications. The filter was fabricated on $3 \mathrm{M} \mathrm{Cu}$-clad217 substrate from Arlon with a $0.76 \mathrm{~mm}$-thick and relative dielectric constant 2.17. Following the design methodology in the preceding section the filter's dimensions were determined to be (in millimeters): $L_{1}=5, L_{2}=13, L_{3}=7, L_{4}=1, L_{5}=3, L_{6}$ $=9, L_{7}=8, L_{8}=1, L_{9}=1, L_{10}=5.5, W_{1}=0.5, S_{1}=1.1, S_{2}=1$, $S_{3}=S_{4}=0.23$, and $W_{2}=2.42$, which corresponds to a characteristic impedance of $50 \Omega$. Simulation was performed using Keysight Technologies' Momentum 3D planar EM simulator based on the method-of-moment. The filter's performance was measured using a scalar network analyzer.

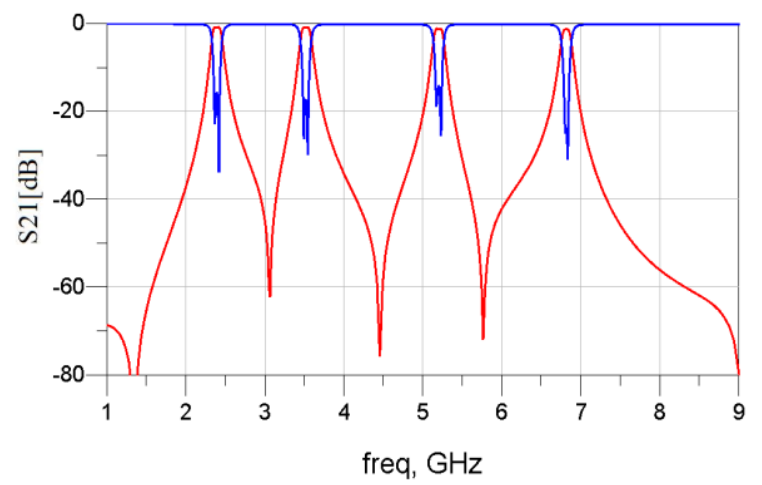

Fig. 4 Simulated insertion-loss and return-loss response of the proposed filter.

Fig. 4 and 5 show the simulation and the measured results, respectively. Referring to the simulated result in Fig. 4, the filter has four passband responses centered at 2.4/3.5/5.2/6.8 $\mathrm{GHz}$ with a high out-of-band rejection. The insertion-loss at the four passbands is less than $0.7 \mathrm{~dB}, 0.74 \mathrm{~dB}, 1.1 \mathrm{~dB}$ and 1.2 $\mathrm{dB}$, respectively. The corresponding return-loss is $32 \mathrm{~dB}, 29$ $\mathrm{dB}, 25 \mathrm{~dB}$ and $30 \mathrm{~dB}$, respectively. The five transmission zeros between the passbands result in the realization of a filter with high selectivity and high isolation between adjacent passbands. There is excellent correlation between the simulation and measured result. The slight discrepancy is attributed to fabrication tolerance.

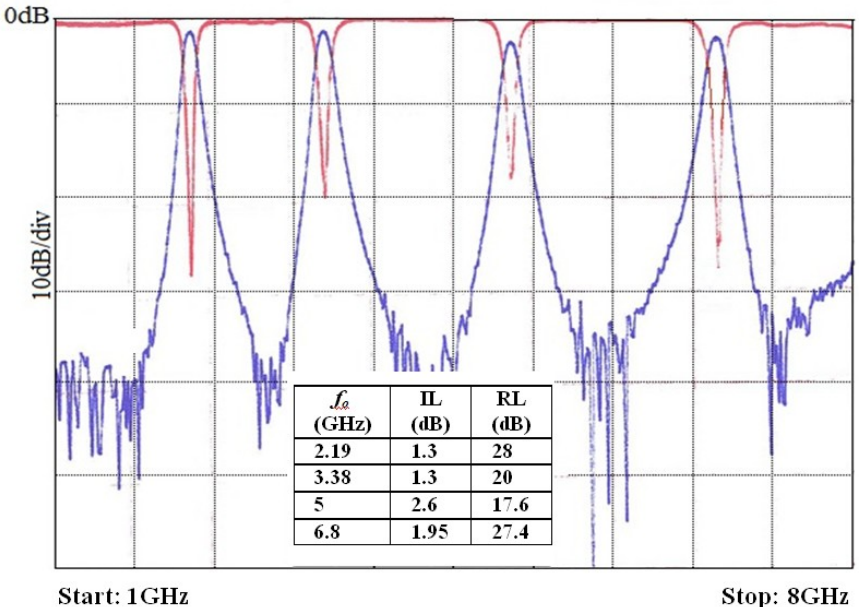

Fig. 5. Measured insertion-loss and return-loss response of the proposed filter.

\section{CONCLUSION}

A highly compact planar quad-band bandpass filter was presented based on stub-loaded resonators. The two pairs of resonators are used to excite quad-mode resonances. The proposed technique provides a sufficient degree of freedom to alter the filter's specifications in terms of centre frequency and bandwidth. Source to load coupling and the resulting two transmission paths are inherently realized to create transmission zeros, resulting in high selectivity and high isolation between the four passbands. The design methodology has been verified through practical implementation of a prototype filter. The filter's performance and compact size makes it attractive for application in multiband wireless communication systems.

\section{REFERENCES}

[1] M. Studniberg, G. V. Eleftheriades, "A quad-band bandpass filter using negative-refractive-index transmission-line (NRI-TL) metamaterials," IEEE Proc. Ant. Propag. Soc. Int. Symp., 2007, pp. 4961-4964.

[2] J.C. Liu, J.W. Wang, B.H. Zeng, D.C. Chang, "CPW-fed dual mode double-square-ring resonators for quad-band filters," IEEE Microw. Wireless Compon. Lett., 20, (3), Mar. 2010, pp. 142-144.

[3] C. Cui, Y. Liu, "Quad-band bandpass filter design by embedding dualband bandpass filter with dual-mode notch elements," Electronics Letters, 50, (23), Nov. 2014, pp. 1719-1720.

[4] J-K. Xiao, Y-F. Zhu, Y. Li, X-W. Li, "Miniature quad-band bandpass filter with passband individually controllable using folded SIR," Electronics Letters, 50, (9), April 2014, pp. 679-680.

[5] H. Liu, B. Ren, X. Guan, P. Wen, Y. Wang, "Quad-band hightemperature superconducting bandpass filter using quadruple-mode square ring loaded resonator," IEEE Trans. Microw. Theory and Tech., 62, (12), Dec. 2014, pp. 2931-2941.

[6] X.Y. Zhang, Q. Xue, "Dual-band bandpass filter using stub-loaded resonators," IEEE Microw. Wireless. Compon. Lett., 17, (8), Aug. 2007, pp. 583-585. 